\title{
Motivos de consulta e hipótesis clínicas explicativas
}

\section{Reasons for consultation and clinical explicative hypothesis}

\author{
Amanda M. Muñoz-Martínez \\ $\&$ \\ Mónica Ma. Novoa-Gómez \\ Grupo de Investigación en Psicología y Salud \\ Pontificia Universidad Javeriana, Colombia
}

(Rec: 4 de mayo de 2011 / Acep: 20 de noviembre de 2011)

\begin{abstract}
Resumen
Los motivos de consulta implican la demanda que hacen los consultantes acerca de problemas que ellos perciben en su vida diaria; en este estudio se investigó sobre las características de estos y las hipótesis explicativas de 61 casos que fueron atendidos por terapeutas desde una perspectiva conductual, en una institución de servicios psicológicos. Los motivos de consulta más frecuentes se relacionaron con comportamientos agresivos (usualmente presentados en los hombres) y respuestas emocionales intensas (casi siempre reportadas por las mujeres). Además, se encontró que los descriptores usados por los consultantes fueron incluidos en las hipótesis explicativas elaboradas por los terapeutas. Se discute la relevancia del motivo de consulta como elemento guía del proceso terapéutico, la implicación de su asociación con las hipótesis explicativas y la importancia de desarrollar otros estudios que pongan en evidencia la relación entre estos y otras variables. Palabras clave: Motivos de consulta, hipótesis explicativa, modelo comportamental, intervención psicológica.
\end{abstract}

\begin{abstract}
Reasons for consultation involve consultant's requests about problems they perceive into their lives. This study researched the characteristics of these reasons for consultation and the explicative hypothesis of 61 cases treated by therapists from a behavioral perspective, within an institution for psychological services. The most frequent reasons for consultation were related to aggressive behaviors (usually presented on men) and intense emotional responses (almost always reported by women). Moreover, we found that the descriptors used by consultants were included on the therapists' explicative hypothesis. We discuss the relevance of reasons for consultation to guide the entire psychotherapeutic process, the implication of its association to the explicative hypothesis and the relevance to carry out other studies that uncover the relation between them and other variables.

Keywords: Reasons for consultation, explicative hypothesis, behavioral model, psychological treatment.
\end{abstract}




\section{Introducción}

Existe un vasto número de publicaciones relacionadas con la terapia psicológica, que en su mayoría han abordado la pertinencia de las intervenciones para el manejo de las problemáticas referidas por los consultantes. Sin embargo, resulta curioso observar que los estudios relacionados con los motivos de consulta y su función dentro del proceso terapéutico son pocos (Quesada, 2004), aunque con frecuencia se afirma que estos implican más que la descripción de los problemas y las razones por las cuales las personas consultan (Kramer, Kleindorfer \& Colarelly-Beatty, 1994; Martínez, 2006; Rodríguez, 2007), en tanto los autores suponen que su seguimiento incrementa el compromiso con la intervención por parte de los consultantes (Calderón et al., 2008).

El interés en el tema ha aumentado en los últimos años. Así, en Colombia se llevó a cabo un estudio al respecto por parte de la Red de Instituciones de Servicios Universitarios de Atención Psicológica -ISUAP- (2007), con el que se buscaba hacer un perfil de los motivos de consulta que se referían con mayor frecuencia en diferentes centros de atención psicológica universitaria en Colombia. Los resultados mostraron que los problemas de comportamiento eran los más frecuentes en la población, seguidos por problemas del estado de ánimo y ansiedad. Sin embargo, los datos utilizados para ello fueron las impresiones diagnósticas iniciales, dadas por los terapeutas en el primer encuentro con los consultantes, dejando de lado las descripciones referidas por ellos mismos y la implicación de las problemáticas en relación con el ajuste a los diferentes contextos.

En un estudio llevado a cabo por Loubat \& Magaña (2005) en un centro de atención psicológica en Chile, se mostró el perfil epidemiológico de los motivos de consulta por tres años, encontrándose que la población asistente reportaba con mayor frecuencia problemas de relación, trastornos de ansiedad, adaptativos y depresivos en orden de ocurrencia. No obstante, los datos analizados correspondían a la clasificación diagnóstica que hacían los terapeutas en el primer encuentro con los consultantes y centraron su atención en la elaboración de un perfil epidemiológico, con base en datos que no hacían referencia directa a los motivos de consulta expresados por los consultantes, alejándose de su objetivo principal.

Lo anterior muestra que las investigaciones en el tema comparten dos aspectos: (a) la escasa consideración sobre el efecto y la forma en que los problemas descritos afectan el funcionamiento de las personas y (b) la equivalencia que hacen los investigadores clínicos entre el motivo de consulta y la clasificación de las problemáticas del consultante en términos de categorías diagnósticas psiquiátricas, derivadas de un primer encuentro con el consultante, que no cumple con los postulados propuestos en el DSM-IV (1994).
De esta manera, los estudios en los que los datos han sido analizados bajo los criterios mencionados resultan insuficientes para establecer las razones por las cuales las personas deciden acudir a los servicios psicológicos, pues no dan cuenta de la demanda psicológica, las conductas problema y el grado de afección de quien consulta.

Al respecto, Martínez (2006) señala que aunque esto puede entenderse por la necesidad que tuvieron en un principio los terapeutas de establecer un diagnóstico de manera rápida, debido a las características propias del contexto en el que se consolidó la práctica clínica psicológica, este es un fenómeno que aún se sigue presentando, como se evidencia en el estudio realizado por Maschi, Schwalbe, Morgen, Gibson \& Violette (2009), en el que se analizó la influencia del género de los adolescentes en el uso de servicios psicológicos. En éste es posible observar cómo los autores utilizaron un inventario que clasificaba a los participantes de acuerdo con un criterio nosológico, para derivar a partir de los puntajes obtenidos por ellos el tipo de abordaje psicoterapéutico.

Ese tipo de aproximaciones puede ser contraproducente para el ejercicio profesional en la actualidad, pues los análisis hechos con base en esa información pueden limitar el desarrollo de políticas de prevención y promoción ajustadas a las necesidades reales de la población y posibilitando el mantenimiento de los comportamientos problema recurrentes que permanecen por períodos extensos y pueden llegar a ser incapacitantes (Ortega, Roca, Iglesias \& Jurado, 2004).

Rodríguez (2007) afirma que algunos de los problemas con el estudio de los motivos de consulta, pueden estar relacionados con las dificultades históricas que ha presentado el término, pues los profesionales han puesto dentro de la misma categorías los síntomas, que provienen de una tradición médica, con las descripciones de algunas formas de conducta problema que señalan los consultantes durante los primeros encuentros con el terapeuta. En relación con ello, la autora señala que para llevar a cabo investigaciones en el tema, es necesario delimitar previamente qué se entiende por motivo de consulta y lo que éste implica. De acuerdo Rodríguez (2007), "el motivo de consulta no es un problema, es una comunicación inicial que denota inquietud y alarma" (p. 245), de tal forma que éste implica descripciones de algunas formas de conducta que son problemáticas, pero no hace referencia a síntomas de una patología subyacente, enmarcando así al motivo de consulta como una guía sobre la que ha de comenzar a indagar el terapeuta, dado que inicialmente hace referencia a las inquietudes del consultante, pero posteriormente podría relacionarse con otro tipo de elementos.

A nivel práctico, algunos autores han hecho investigaciones coherentes con lo planteado por Rodríguez (2007), partiendo de las descripciones hechas por las personas en el momento inicial de la consulta, sin hacer uso de los diagnósticos referidos por los terapeutas. Quesada (2004), en la 
Argentina, clasificó los casos en términos de los elementos mencionados por los consultantes, y estableció cuatro categorías que no eran mutuamente excluyentes, con el fin de comprender mejor el fenómeno, éstas fueron: problemáticas sociales, problemas familiares, problemas de pareja, problemáticas con la sexualidad y síntomas somáticos.

Dentro de los problemas sociales se incluyeron descripciones relacionadas con la elección vocacional, la interacción con otras personas implicadas en sus diferentes contextos y en general aquellos elementos asociados con lo que se espera socialmente de las personas. La categoría de problemas familiares incluyó las descripciones en las que se referían las relaciones con los miembros del núcleo familiar biológico y extenso. En las problemáticas de pareja se tuvieron en cuenta los elementos asociados al establecimiento, mantenimiento e insatisfacción con las relaciones afectivas. En las dificultades en la sexualidad como inhibiciones, disfunciones y orientación y elección sexual. Finalmente, en la categoría de síntomas somáticos se incluyeron las respuestas relacionadas con reacciones biológicas problemáticas para el funcionamiento (Quesada, 2004).

Los resultados mostraron que en los motivos de consulta se hacía referencia con mayor frecuencia a los problemas en el contexto familiar, seguido por las dificultades en el contexto social, de pareja, en la sexualidad y finalmente las problemáticas relacionadas con respuestas somáticas, caracterizando los motivos de consulta de una mejor forma dado que se analizaron guardando coherencia con lo que implican ellos y alejándose de un modelo médico para el abordaje de los problemas psicológicos (Quesada, 2004).

Así pues, los datos de dicha investigación permitieron establecer de forma más ajustada los elementos mencionados en los motivos de consulta. Sin embargo, no muestra cómo estos se enlazan con las hipótesis explicativas que los clínicos se hacen de cada caso y finalmente sí esto podría estar relacionado con la efectividad terapéutica. Pues aunque la identificación de los elementos constitutivos del motivo de consulta resulta útil para la práctica clínica, dentro del modelo idiográfico y conductual esto no es suficiente para llevar a cabo un proceso terapéutico, ya que esta postura plantea que las intervenciones han de derivarse teniendo en cuenta los elementos de los cuales la conducta es función (Bergner, 1998; Haynes, Leisen \& Blaine, 1997; Iwata \& Worsdell, 2005; Northup et al.,1991; Reep \& Horner, 2000 citado en Reep \& Horner, 2000; Virués-Ortega \& Haynes, 2005).

Debido a ello, es importante no sólo el establecimiento de los repertorios o problemas conductuales problemáticos, sino también que las hipótesis o formulaciones de los casos tengan un bajo nivel de inferencia acerca de la forma en que se asocian los comportamientos problema con las variables del contexto particular de los consultantes (Eells, 2001; Eells, Kendjelic \& Lucas 1998; Mumma \& Mooney, 2007; Mumma \& Smith, 2001) que pueden o no estar asociadas con el motivo de consulta inicial (Caycedo, Ballesteros \& Novoa, 2008; Muñoz-Martínez \& Novoa-Gómez, 2010).

En relación con lo expuesto el presente estudio busca caracterizar los motivos de consulta de las personas atendidas por terapeutas desde una perspectiva conductual, para establecer qué tipo de asociación existe entre las descripciones hechas en los motivos de consulta y los elementos mencionados en las hipótesis generadas para explicar los casos y entre las hipótesis que tienen elementos en común con los motivos de consulta y el éxito terapéutico en esta población.

\section{Método}

\section{Diseño}

Estudio retrospectivo con una aproximación multimétodo cuantitavo-cualitativo y análisis de datos descriptivo y factorial.

\section{Participantes}

Se revisaron 222 historias clínicas llevadas a cabo por terapeutas en el enfoque comportamental en el periodo comprendido entre los años 2005 al 2008, en un centro universitario de atención psicológica de la ciudad de Bogotá.

Las historias fueron clasificadas y se seleccionaron 61 teniendo en cuenta como criterio de inclusión contener dentro del reporte las narrativas del motivo de consulta y de las hipótesis explicativas o formulaciones explicativas del caso.

\section{Instrumentos}

La información de las historias clínicas fue codificada en una base de datos en el programa Microsoft Excel en la que se incluían los datos demográficos del consultante, número de la historia clínica, sexo, escolaridad y edad. También contenía datos propios del proceso terapéutico como si el caso había finalizado exitosamente, si se incluía la hipótesis explicativa y la formulación de caso y si tenía elementos en común con el motivo de consulta. Posteriormente se creó una base de datos en el software de análisis estadístico SPSS v.17 a través del cual se llevó a cabo el análisis descriptivo de los datos y se establecieron las asociaciones entre ellos.

Sumado a lo anterior, se transcribieron las hipótesis funcionales y los motivos de consulta de los 61 casos seleccionados, con el fin de efectuar el análisis de las narrativas por medio del software de análisis de datos textuales ATLAS ti v.5.0 y así establecer las asociaciones entre esos dos elementos.

Además se utilizó el programa SPAD (Système Portable pour L'Analyse des Donees Textuelle) para llevar a cabo el análisis estadístico de los datos cualitativos, llevando a cabo el análisis de correspondencias simples entre el sexo y la escolaridad de los consultantes con las narrativas de 
los motivos de consulta y la identificación de los elementos léxicos representativos para cada variable.

\section{Procedimiento}

Inicialmente se revisaron y seleccionaron las historias clínicas de los casos atendidos por terapeutas dentro del modelo conductual, archivadas en un centro universitario de atención psicológica. Tras este proceso se identificaron 222 historias clínicas y se descartaron 161, dado que no cumplían con los criterios de inclusión para el análisis de datos. Después de ello se transcribieron los motivos de consulta y las hipótesis funcionales o formulaciones de los casos consignadas por los terapeutas.

Posteriormente se concretó el análisis cualitativo de las narrativas o verbatims de las hipótesis funcionales y los motivos de consulta transcritos. Estas narrativas hacían referencia a las descripciones puntuales de los textos contenidos en las historias clínicas, los cuales permitieron el ordenamiento conceptual que implicaba la organización de los datos en categorías con el fin de establecer un sistema de clasificación de acuerdo con las propiedades determinadas en el marco conceptual de la investigación (Strauss \& Corbin, 2002).

Siguiendo lo anterior, se utilizó la herramienta de análisis cualitativo Atlas ti 5.0 para establecer las asociaciones entre los términos mencionados en los verbatims de los motivos de consulta con los incluidos en las hipótesis explicativas, permitiendo observar la forma en que los elementos de unas y otras se tenían en cuenta para describir y explicar los casos. Además, se elaboraron redes conceptuales (redes estructurales), que son representaciones gráficas del ordenamiento conceptual y que permitieron visualizar la forma en la que se relacionaban los motivos de consulta con las hipótesis explicativas.

Finalmente se llevó a cabo el análisis de los datos a través del SPAD. Se identificaron los elementos léxicos representativos del motivo de consulta de acuerdo con el sexo y el nivel escolar y el análisis de correspondencias simples para identificar los factores que explicaban la distribución de las narrativas para los dos factores que agrupaban la mayor parte de los datos.

\section{Resultados}

El análisis de los datos socio-demográficos no mostraron diferencias importantes relacionadas con el sexo, pues el $59 \%$ de los casos eran hombres y el $41 \%$ mujeres. Con respecto a la edad de los consultantes (Figura 1), se observó que ésta se distribuía entre los 4 y los 55 años, con una media de 20.46 años, una mediana de 15 años y una moda de 8 años, con una desviación estándar de 14. Estos datos permiten decir que en la mayoría de los casos la asistencia a consulta dependía en mayor medida de personas responsables de los consultantes que de ellos mismos, puesto que buena parte de la muestra tenía edades inferiores a la mayoría de edad.

Los hallazgos relacionados con la escolaridad (Figura 2) se analizaron con respecto al último año cursado por los consultantes. Los datos mostraron que la mayoría de las personas que asistieron a consulta durante ese periodo se encontraban cursando educación básica primaria o básica secundaria y los que menos asistieron eran personas con un nivel escolar de primaria completa. Estos datos se

Figura 1. Distribución de los consultantes de acuerdo con la edad.

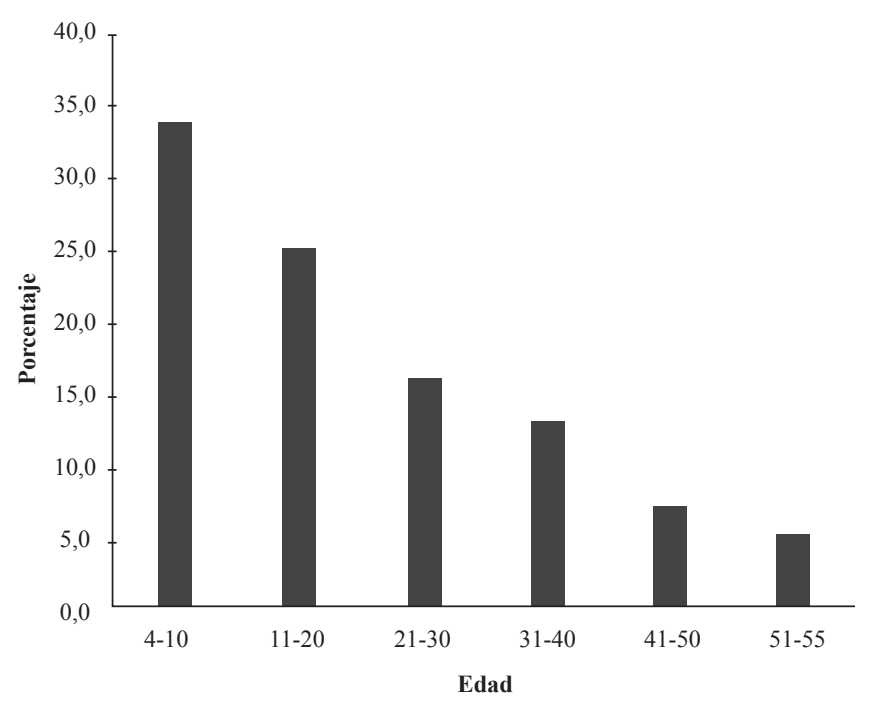


Figura 2. Distribución de los consultantes de acuerdo con la escolaridad.

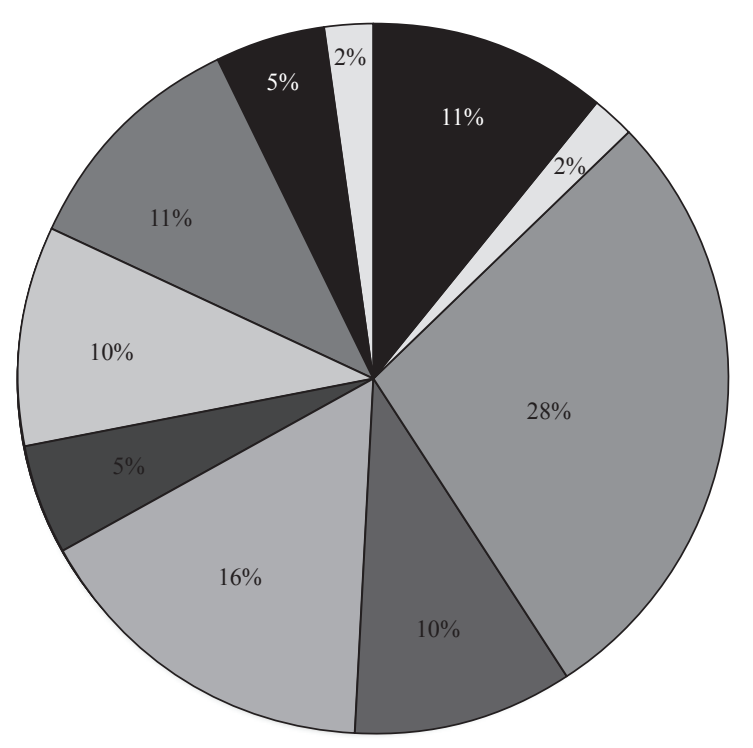

Preescolar en curso

Primaria

Primaria en curso

Secundaria

Secundaria en curso

Secundaria incompleta

Técnico

Universitaria

Universitaria en curso

Universitaria incompleta

Figura 3. Porcentaje de historias clínicas respecto al éxito terapéutico.

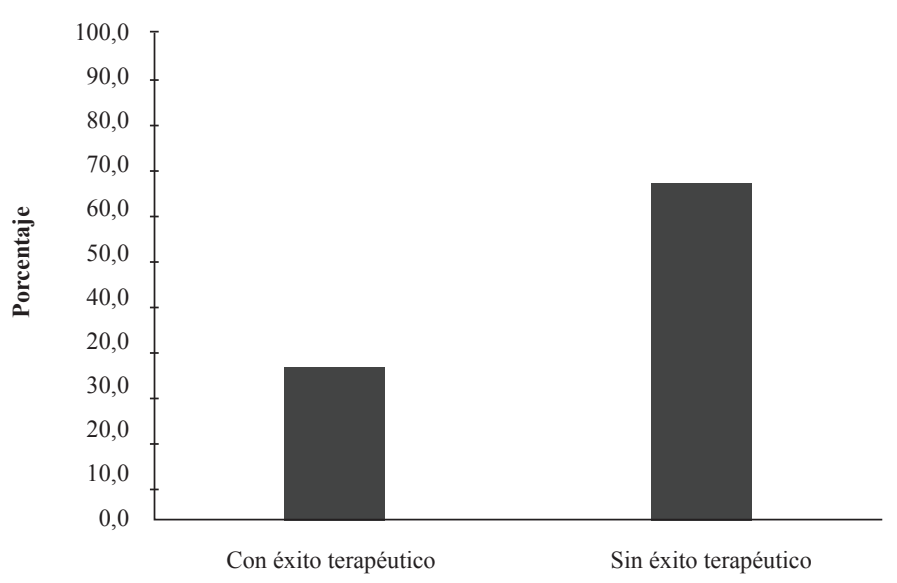

relacionan con la distribución de la edad, pues la mayoría de ellos se encontraban por debajo de los 20 años, por lo que buena parte de la muestra estaba distribuida entre aquellos que cursaban estudios de primaria y secundaria.

En relación con los datos de los elementos implicados en el proceso terapéutico se encontró que el $31.1 \%$ de estos fueron exitosos, en términos del cumplimiento de los objetivos terapéuticos y/o la eficacia de la intervención (Figura 3). Además se encontró el que el $47.5 \%$ de los terapeutas presentaban dentro de las historias clínicas su conceptualización del caso (en un formato específico de formulación), mientras el 52.5\% no (Figura 4). No obstante, se observó que el 96.7\% de las historias incluían en su registro una hipótesis explicativa del caso (Figura 5), lo que permite 
Figura 4. Porcentaje de historias clínicas en las que se hace explícita la formulación de caso.

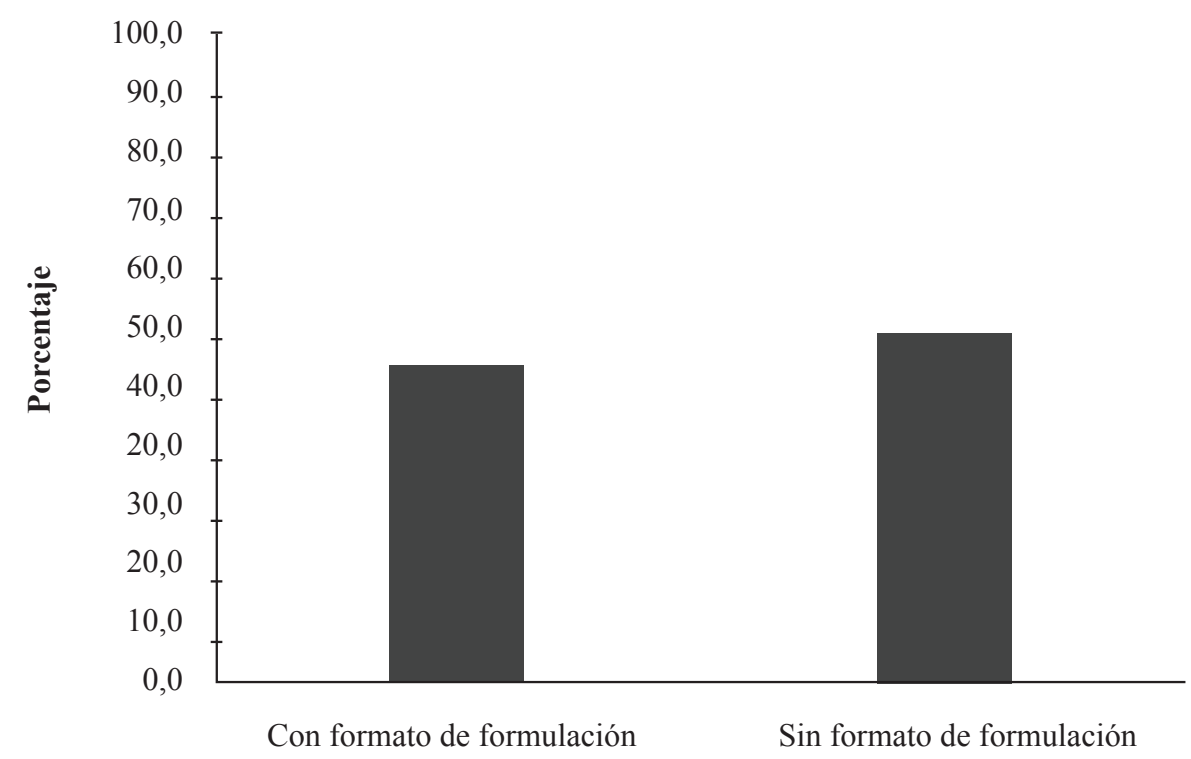

Figura 5. Porcentaje de historias de acuerdo con la hipótesis explicativa.

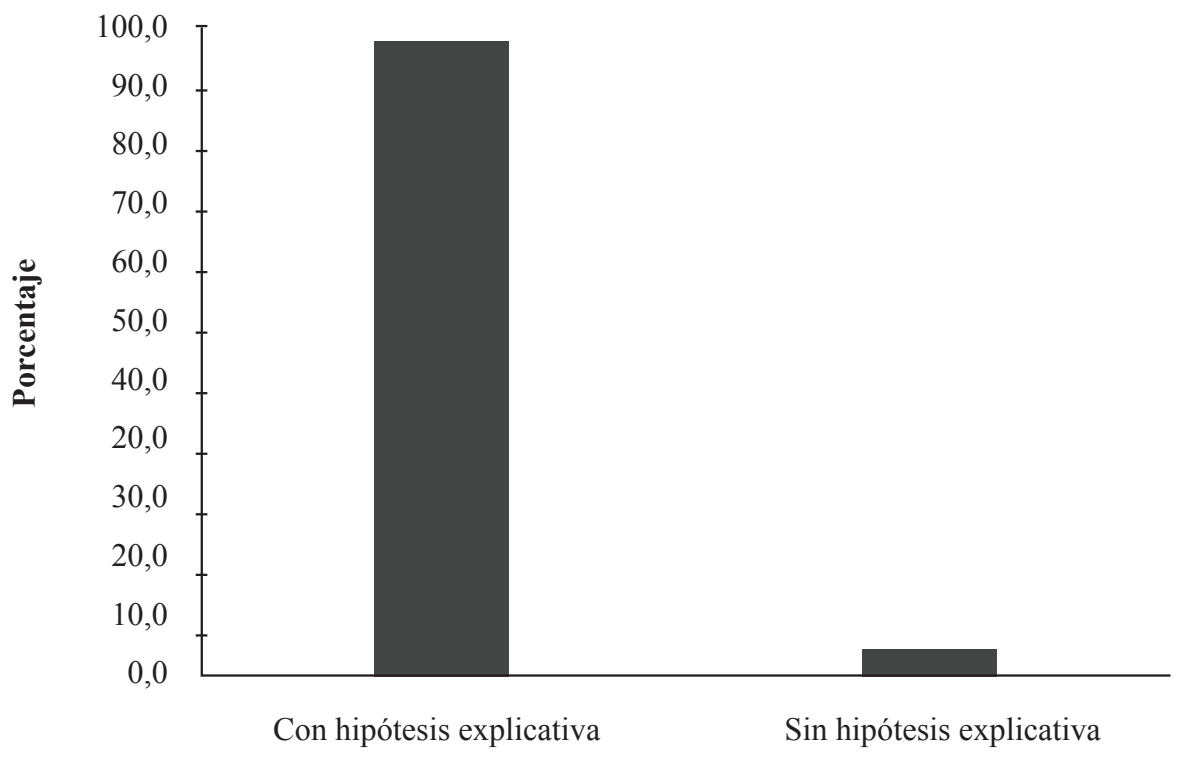

suponer que a pesar de no incluir un modelo de formulación determinado, los psicólogos tendieron a hacer hipótesis en las que consideraron varios elementos relacionados con el momento en el que las personas llegaban a consulta y la historia del consultante para guiar el proceso terapéutico.

En cuanto a la inclusión de los descriptores de los motivos de consulta en las hipótesis explicativas o en las formulaciones, se observó que en el 93.4\% de los casos las hipótesis contenían elementos de los motivos de consulta, siendo mucho mayor que aquellos en los que no se asociaban estos dos elementos (Figura 6).
Al respecto, se encontró que en los motivos de consulta, las 61 personas hicieron alusión a 38 tipos de problemas, con 122 descriptores en total, dado que en múltiples ocasiones los consultantes reportaban de forma concurrente más de un elemento problemático, esto posiblemente se debe a que las problemáticas abarcan más de una topografía de comportamiento y pueden involucrar más de un contexto, situación y/o persona al mismo tiempo.

Las problemáticas que se mencionaron un mayor número de veces fueron los comportamientos agresivos y las respuestas emocionales, referidas en 11 ocasiones, seguidas 
Figura 6. Porcentaje con el que se incluyen descriptores del motivo de consulta (MC) en las hipótesis explicativas .

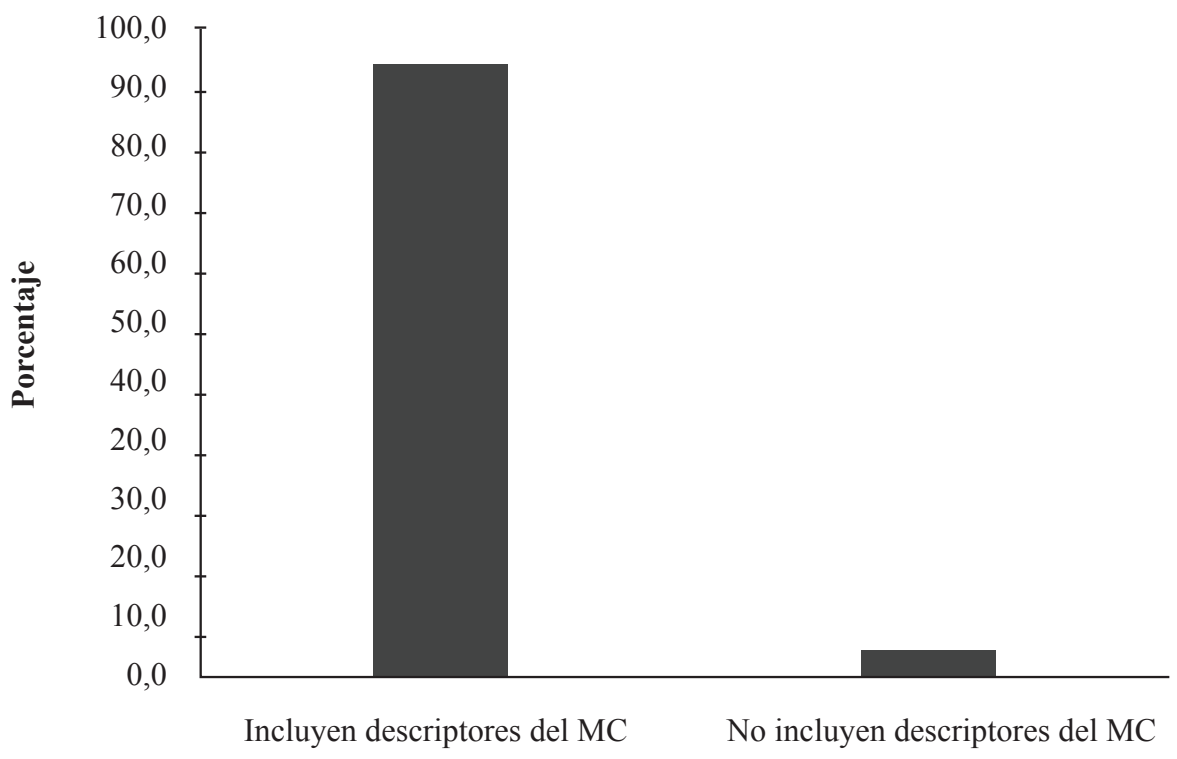

por las dificultades en la interacción social y en las relaciones familiares, mencionadas 10 veces cada una. Mientras que las dificultades que se reportaban en menor medida, dado que sólo fueron mencionadas en una ocasión, fueron el abandono de actividades cotidianas, el afrontamiento al abuso sexual, el ajuste a situaciones novedosas o estresantes, el desempeño escolar, las amenazas de suicidio, entre otros.

Con respecto a las hipótesis funcionales se encontró que los terapeutas utilizaron 43 tipos de componentes para hacer la descripción de los problemas de los consultantes, los cuales fueron mencionados 143 veces (Tabla 2). El tipo de descriptor más utilizado fue el de comportamiento agresivo mencionado en 15 ocasiones, seguido por las dificultades en la interacción social que se mencionó en 12. Algunos de los elementos que se utilizaron en sólo una ocasión fueron las alteraciones en el sueño, anhedonia, comportamiento relacionado con la pérdida de reforzadores y dificultades en la concentración.

El análisis de correspondencias mostró que efectivamente los datos se agrupaban en dos factores: sexo y nivel escolar (último nivel alcanzado por los consultantes). En la Tabla 1 se puede ver como el género contribuía en una mayor medida en el factor 1 y el nivel de escolaridad en el 2. Se puede observar que en el factor 2 las variables con una mayor contribución fueron el universitario incompleto y la secundaria incompleta, como últimos niveles alcanzados.
En el plano factorial (Figura 7) se presentan las variables determinantes de los factores y la representación de los datos textuales de los motivos de consulta, que están constituidos por las 50 palabras con sentido semántico más contributivas para los factores 1 y 2 . La distribución de los datos permite ver que hay dos tipológicas de la demanda psicológica en los motivos de consulta. El primero se dio en relación con el sexo de los consultantes (elementos seleccionados en los rectángulos con línea continua) y el segundo se constituyó en torno al último nivel escolar alcanzado (elementos seleccionados en los rectángulos con línea discontinua).

De esta manera, en el plano se puede observar que las mujeres en sus motivos de consulta mencionaban palabras como quiero, pienso y siento, que hacían referencia a elementos de su propia conducta, también utilizaban palabras como relación, pareja, esposo, padres e hija y se encontraron otras como miedo, llorar y preocupa. Así, los motivos de consulta de las mujeres estaban caracterizados por demandas relacionadas con sus interacciones con su pareja e hijos, palabras que mostraban que ellas eran las que demandaban el servicio y hacían referencia a respuestas emocionales.

Por otro lado, en el caso de los hombres, generalmente las palabras asociadas hacían referencia a otras personas y a las formas de comportamiento (por ejemplo, inquieto, hiperactivo, agresividad) que otros describían; además, se 
Tabla 1. Coordenadas y contribuciones de las frecuencias para los ejes 1 y 2 .

\begin{tabular}{lcccccccc}
\hline \multirow{2}{*}{ Modalidad } & \multicolumn{2}{c}{ Frecuencias } & \multicolumn{2}{c}{ Coordenadas } & \multicolumn{2}{c}{ Contribuciones } & \multicolumn{2}{c}{ Cosenos Cuadrados } \\
& P. Rel & Disto & 1 & 2 & 1 & 2 & 1 \\
\hline Hombre & 30.93 & 0.11 & -0.26 & 0.04 & 13.9 & 0.4 & 0.63 & 0.01 \\
Mujer & 19.07 & 0.28 & 0.42 & -0.06 & 25.5 & 0.7 & 0.63 & 0.01 \\
Preescolar en curso & 8.40 & 0.68 & -0.40 & -0.12 & 9.0 & 1.2 & 0.23 & 0.02 \\
Primaria & 1.29 & 4.96 & 0.25 & -0.59 & 0.5 & 4.3 & 0.01 & 0.07 \\
Primaria en curso & 13.19 & 0.48 & -0.36 & -0.05 & 11.5 & 0.3 & 0.27 & 0.00 \\
Secundaria & 3.59 & 1.77 & 0.45 & 0.23 & 4.9 & 1.9 & 0.12 & 0.03 \\
Secundaria en curso & 7.88 & 0.72 & -0.26 & -0.12 & 3.7 & 1.0 & 0.10 & 0.02 \\
Secundaria incompleta & 1.45 & 5.87 & 0.70 & 1.42 & 4.7 & 28.4 & 0.08 & 0.34 \\
Técnico & 2.75 & 2.86 & 0.81 & -0.66 & 12.0 & 11.8 & 0.23 & 0.15 \\
Universitaria & 7.07 & 0.93 & 0.49 & -0.17 & 11.2 & 1.9 & 0.26 & 0.03 \\
Universitaria en curso & 3.45 & 2.06 & 0.50 & 0.40 & 5.9 & 5.5 & 0.12 & 0.08 \\
Universitaria incompleta & 0.93 & 9.91 & -0.18 & 2.18 & 0.2 & 42.6 & 0.00 & 0.48 \\
\hline
\end{tabular}

Figura 7. Plano factorial de las variables género y nivel escolar.

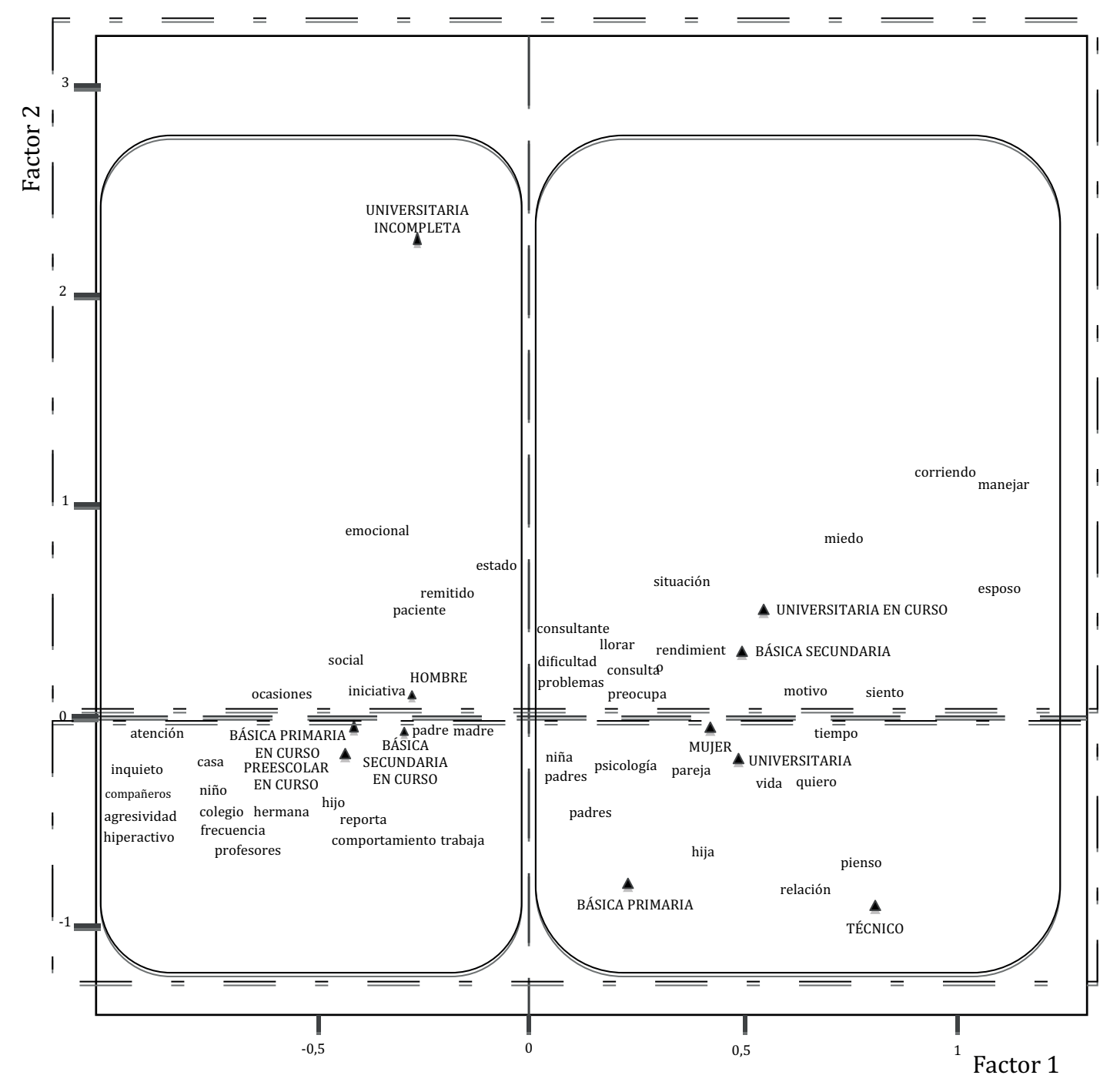


Tabla 2. Segmentos representativos de los motivos de consulta.

\begin{tabular}{ll}
\hline Sexo & \multicolumn{1}{c}{ Formas léxicas representativas } \\
\hline Hombre & El niño tiene comportamientos que son inadecuados en el colegio en el que estudia, no pone atención y roba junto con sus \\
& compañeros. Además, en el colegio reportan haberlo visto junto con otros compañeros consumiendo drogas. \\
Mujer & Es mi enfermedad, yo me he enfermado varias veces, primero me diagnosticaron trastorno maniaco depresivo, que ahora \\
& se llama trastorno afectivo bipolar tipo I y la última doctora con la que estuve me dijo que yo no era eso, sino que era \\
& esquizo-afectiva. El doctor que me atendió, uno flaquito, blanquito y buen mozo, me dijo que yo ya estaba bien de \\
& mi enfermedad y que me remitía con usted, porque a mí me da mucho miedo volverme a enfermar, yo pienso y me \\
& imagino que mi comportamiento no es el adecuado, creo que a mí la gente no me aprecia, sino que sienten lástima por mi \\
& enfermedad y se burlan de mí.
\end{tabular}

mencionaban palabras como remitido y reporta. En relación con lo anterior, la asistencia a consulta por parte de los hombres podría darse por la búsqueda de ayuda que hacen los que interactúan con ellos y no porque ellos mismos la soliciten.

Muestra de lo expuesto previamente son las formas léxicas representativas de los motivos de consulta de hombres y mujeres, las cuales hacen referencia a los segmentos textuales que ilustran de mejor forma el factor 1 , pues están constituidos por buena parte de las palabras que tienen una mayor contribución en éste (Tabla 2).

Respecto al segundo factor se puede observar que las dos variables que tienen una mayor contribución en él se encuentran aisladas y las palabras claves en la distribución se encontraban lejos de ellos. Sumado a esto, se puede ver como las variables del sector superior en el plano (determinado por las personas que habían alcanzado un nivel educativo básico o que no tenían estudios superiores) implicaban las palabras miedo, emocional, llorar, rendimiento, manejar y preocupa, que se relacionan con problemas de tipo emocional que suelen afectar el propio funcionamiento en los diferentes contextos. Mientras en el sector inferior del plano se puede ver que palabras contributivas de éste hacen referencia a otras, el rol de las personas y su etiqueta, por lo que podría decirse que para aquellas personas que tienen un nivel educativo superior o que están cursando alguno de los niveles de educación básica (es posible que ellos hayan sido llevados a consulta por sus cuidadores), los motivos de consulta se encuentran relacionados con el tipo de interacción que tienen con los otros.

Con respecto al uso de los descriptores del motivo de consulta en las hipótesis explicativas, se halló que en cuatro de los 61 casos (C) estudiados no se relacionaban entre sí (Tabla 3). Cabe señalar que sólo uno de estos casos tuvo éxito terapéutico, el cual contenía dentro de su historial la formulación de caso, aunque en ella no se describía la hipótesis funcional, ni el tipo de intervención que se llevó a cabo.

La Figura 8 muestra como en el caso 22 (C22) la narrativa del motivo de consulta se refiere a dificultades en las relaciones afectivas y en la hipótesis explicativa se describe el problema como dificultades en la regulación emocional, sin retomar los elementos mencionados inicialmente por el consultante y como estos derivan en la hipótesis.

Finalmente se realizó el análisis de la asociación entre el éxito terapéutico y las hipótesis explicativas que contenían en sus descripciones elementos de los motivos de consulta, a través de la prueba ji-cuadrado, la cual mostró que la asociación entre estos dos no fue significativa, pues se obtuvo un grado de significación de 0.78 con una $\mathrm{p}<0,05$.

Tabla 3. Motivos de consulta e hipótesis explicativas en las que no hay relación entre los elementos utilizados para describir el problema.

\begin{tabular}{lll}
\hline \multicolumn{1}{c}{ Consultante } & \multicolumn{1}{c}{ Motivo de consulta } & \multicolumn{1}{c}{ Hipótesis explicativa } \\
\hline C5 & Problemas en las habilidades cognoscitivas superiores. & $\begin{array}{l}\text { Dificultades en la interacción social. } \\
\text { Problemas en los repertorios de autoconocimiento. }\end{array}$ \\
C18 & $\begin{array}{l}\text { Sufrimiento. } \\
\text { Manejo de la enfermedad. }\end{array}$ & $\begin{array}{l}\text { Dificultad en la interacción social. } \\
\text { Pensamientos disfuncionales. } \\
\text { Respuesta de evitación. } \\
\text { Respuestas emocionales. }\end{array}$ \\
C22 & Dificultades en las relaciones afectivas. & Dificultades en la regulación de la respuesta emocional. \\
C24 & Dificultades en el estado de ánimo. & Dificultades en el autocontrol.
\end{tabular}


Figura 8. Caso en el que no se evidencia relación entre los descriptores del problema en el motivo de consulta y los usados en la hipótesis funcional.

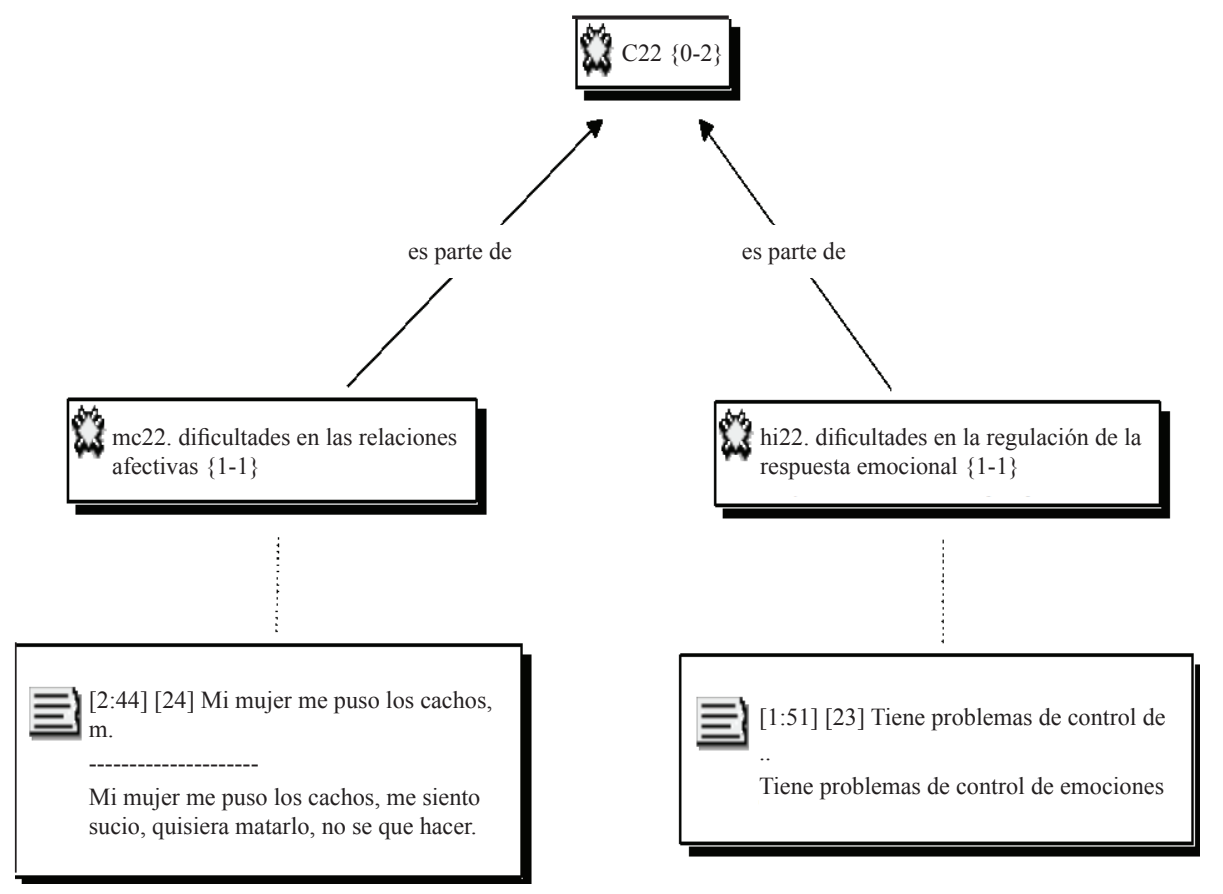

\section{Discusión}

La presente investigación permitió observar el tipo de motivos de consulta que referían las personas asistentes a un centro de atención psicológica sin acudir a categorías diagnósticas, para caracterizar el fenómeno a partir del análisis cualitativo y cuantitativo de los descriptores referidos por los consultantes. Esto es importante en la medida que permite ver con un menor grado de inferencia los problemas que aquejan a las personas que asisten a consulta, partiendo de una visión idiográfica del fenómeno (Iwata \& Worsdell, 2005).

Así, los resultados muestran que el motivo de consulta que refieren con mayor frecuencia los consultantes es la presentación de comportamientos agresivos, seguido de las respuestas emocionales intensas, contrario a lo encontrado en el estudio de Quesada (2004), en donde se vio que el motivo de consulta más frecuente estaba relacionado con aspectos implicados en el contexto familiar que hacían referencia a dificultades en las relaciones entre los padres o entre los padres y los hijos. Es posible que dichas diferencias se relacionen con que en ese estudio la mayoría de consultantes había sido mujeres y, como se mostró en la presente investigación, los motivos de consulta de las mujeres tendían a hacer referencia a problemáticas relacionadas con su familia y la interacción con ellas.

Otro elemento en el que se difiere con el estudio de Quesada (2004) se relaciona con los problemas somáticos, que tenían la frecuencia más baja de ocurrencia, mientras en este estudio ocupó el segundo lugar en frecuencia de presentación. Esto puede explicarse en términos de las características propias del país en donde existen variables contextuales tales como la violencia intrafamiliar y los problemas de seguridad en general, que podrían facilitar la presentación de este tipo de dificultades en la población que asiste a los centros de atención psicológica.

En relación con las variables de tipo sociodemográfico se logró establecer que las mujeres que asistían a consulta referían con mayor frecuencia problemas en su interacción con personas cercanas de su contexto familiar y solían hacer uso de palabras referidas a su propia conducta y demanda, mientras que en los hombres las palabras que contribuían más al factor se referían a la queja que otros tenían de su comportamiento y al desajuste que ello generaba. Esto permite ver la importancia de relacionar las variables sociodemográficas con los datos textuales de los motivos de consulta, para tener una mejor perspectiva que le dé un contexto a estos, y abarcar más adecuadamente la complejidad del fenómeno.

Los análisis también muestran varios aspectos que pueden ser importantes para la investigación en el tema que sobrepasan la caracterización epidemiológica como la hecha por la ISUAP (2007) y Loubat \& Magaña (2005). Pues este estudio permite ver las diferencias en el tipo de demandas que hacen mujeres y hombres e incluso entre las personas de acuerdo con el último nivel educativo alcanzado, lo que lleva a pensar en varias implicaciones, la primera de ellas es el papel de las variables de tipo cultural 
que puedan estar relacionadas con que hombres y mujeres difieran en sus demandas, la segunda es la posibilidad que otro tipo de variables como el estrato socioeconómico o el lugar de procedencia (zona rural o urbana) puedan influir en la forma en la que las personas hacen uso de los servicios psicológicos y por último la implicación de terceros en la valoración de los comportamientos como problemáticos y cómo eso puede facilitar o interferir en el compromiso con los procesos psicoterapéuticos.

Debido a lo anterior, se recomienda para futuras investigaciones incluir datos relacionados con los elementos mencionados y con los factores históricos del período en el que se dieron las consultas, con el fin de determinar si estos se asocian de alguna manera con los motivos de consulta, con el objetivo de realizar programas de prevención encaminados a disminuir el impacto de tales variables en la población (OMS, 2005; Pradilla, Rueda, Salazar, Vélez $\&$ Muñoz, 2007).

Con respecto a las asociaciones entre las descripciones del problema en el motivo de consulta y aquellas incorporadas en las hipótesis explicativas o en la formulación de caso, el análisis de las narrativas permitió ver que 57 de los 61 casos contenían elementos dentro de sus descripciones que relacionaban estos dos, mostrando que en la mayoría de ocasiones los terapeutas tienen en cuenta los reportes iniciales de los consultantes como uno de los ejes centrales que dan una dirección en el ejercicio del psicólogo. Esto guarda coherencia con lo propuesto por la perspectiva conductual en la aproximación que se hace de los casos, partiendo de una visión idiográfica como parte importante en el análisis funcional del comportamiento (Haynes et al., 1997). El análisis de las narrativas permitió ver que en la mayoría de ocasiones los terapeutas tienen en cuenta las descripciones de los consultantes al iniciar los procesos terapéuticos y aunque recogen otros elementos con el paso del tiempo, al momento de llevar a cabo hipótesis alrededor de ellos, estos son retomados.

Empero, aunque los resultados muestran que los elementos que describen la problemática en el motivo de consulta $\mathrm{y}$ aquellos mencionados en las hipótesis funcionales suelen ser similares, también evidencian que estos no tienen una asociación con la efectividad terapéutica, pues en la mayoría de los casos estudiados, exitosos o no, se daba un seguimiento en los elementos referidos en el motivo de consulta, por lo que esta variable no parece ser determinante para garantizar el éxito de la intervención, contrario a lo afirmado por algunos autores (Calderon et al., 2008; Porcel, 2005). Estos resultados no permiten ver cómo este factor puede estar asociado al compromiso terapéutico como lo aseguran dichos estudios, por lo que podría ser un aspecto a indagar en otras investigaciones que estén dirigidas a entender los factores implicados en la relación terapéutica, pues aunque no se asocia directamente con el éxito, puede que sí facilite que la interacción terapeuta-consultante sea sólida y confiable, pero este es un aspecto que habrá de retomarse en otros estudios.

Sumado a ello, los resultados muestran cómo las hipótesis funcionales incluían un mayor número de elementos en la descripción de la problemática, en comparación con los motivos de consulta, lo que puede estar relacionado con que éstas son llevadas a cabo con el fin de sintetizar la información recogida durante el proceso de evaluación (Muñoz-Martínez \& Novoa-Gómez, 2010), incluyendo elementos que no son mencionados en una primera instancia por el consultante, pero que se hacen evidentes al hacer uso de los diferentes métodos de evaluación (Avendaño, 2001; Solhan, Trull, Jahng \& Wood, 2009) y del análisis funcional que se ejecuta tras la exploración de la información desde la perspectiva conductual (Haynes et al., 1997).

En cuanto a los casos en los que no se observó relación entre los verbatims de los motivos de consulta y las hipótesis explicativas, se vio que sólo uno de ellos fue exitoso y que éste había sido formulado con un determinado protocolo. Es posible que en dicho caso la formulación haya sido un elemento relevante para dar cuenta de la problemática, por lo que se puede decir que esta metodología resulta útil para los terapeutas al ayudarlos a esclarecer los problemas de los consultantes y determinar la función de estos en la vida de ellos, siendo éste uno de los objetivos fundamentales de esta metodología (Hayes \& Hass, 1988; Haynes et al., 1997; Muñoz-Martínez \& Novoa-Gómez, 2010).

A manera de conclusión, se puede señalar que el presente estudio permitió ver de forma más descriptiva los motivos de consulta que se refieren en mayor medida en el centro de atención psicológica, como una alternativa a otras investigaciones desarrolladas en el tema (ISUAP, 2007; Loubat \& Magaña, 2005), permitiendo establecer los elementos definitorios de las demandas de los servicios psicológicos en relación con algunas variables demográficas de los consultantes, lo que da una visión más amplia del fenómeno y muestra los aspectos diferenciales entre grupos que habrían de tenerse en cuenta en las políticas de prevención de problemas psicológicos y promoción de conductas saludables, evidenciando la importancia de hacer una evaluación idiográfica del fenómeno (Bergner, 1998; Haynes et al., 1997; Iwata \& Worsdell, 2005; Northup et al.,1991; Reep \& Horner, 2000 citado en Reep \& Horner, 2000; Virués-Ortega \& Haynes, 2005).

Sin embargo, cabe destacar que los datos de la muestra son pequeños en comparación con la población total que asiste no sólo al centro en que se llevó a cabo el estudio, sino en el total de la población que acude a este tipo de servicios. Puede decirse que en principio la metodología utilizada y los datos encontrados son una forma interesante de acercarse a este fenómeno, y abre la posibilidad de realizar más estudios en el área para derivar programas que tengan un mayor impacto, partiendo de datos que se ajusten mejor a las demandas de la población. 
En cuanto al ejercicio psicoterapéutico, los resultados permiten ver que aunque aparentemente los motivos de consulta son una guía importante para los terapeutas al momento de analizar los casos, no son un factor que ayude a garantizar intervenciones exitosas, por lo que es vital trascender este aspecto y hacer énfasis en la realización de investigaciones que identifiquen otras variables que tengan un mayor alcance, con el fin de mejorar la atención psicológica y esclarecer el lugar del seguimiento del motivo de consulta dentro de la práctica clínica.

\section{Referencias}

American Psychiatric Association. (1994). Diagnostic and Statistical Manual of Mental Disorders. 4th ed. Washington, DC: American Psychiatric Press, Inc.

Avendaño, C. (2001). La entrevista clínica, consideraciones clínicas en torno a la mirada terapéutica. Revista Electrónica de Psicología Iztacala. 4. Recuperado en enero 28 de 2009, disponible en http://74.125.113.132/ search?q=cache:VDDzhx4o36QJ:www.iztacala. unam.mx/carreras/ psicologia/psiclin/vol4/vol4art4.html+LA+ENTREVISTA+CL\%C3\% 8DNICA+CONSIDERACIONES+EN+TORNO+A+LA+MIRADA+T ERAPEUTICA+link\&cd=1\&hl=es\&ct=clnk\&gl=es.

Bergner, R. (1998). Characteristics of optimal clinical case formulations. American Journal of Psychotherapy, 52, 287-301.

Calderón, A., De la Cerda, P., Eitel, U., González, V., Klaber, J., Rodríguez, F., \& Vicuña, S. (2008). ¿Qué sucede con los pacientes que abandonan tratamiento? Seguimiento a través de aplicación de encuesta telefónica y OQ 45.2. Recuperado en febrero 06 de 2010, disponible en http:// udd.altavoz.net/psicologia/spi/index2.htm.

Caycedo, C., Ballesteros, B., \& Novoa, M. (2008). Análisis de un protocolo de formulación de caso clínico desde las categorías de bienestar psicológico. Universitas Psychologica, 7, 229-248.

Eells, T, Kendjelic, E \& Lucas, C. (1998). What's in a case formulation? Development and use of a content coding manual. The Journal of Psychotherapy Practice and Research, 7, 144-156.

Eells, T. (2001). Update on psychotherapy case formulation research. The Journal of Psychotherapy Practice and Research, 10, 277-281.

Hayes, S., \& Haas, J. (1988). A reevaluation of the concept of clinical significance: goals, methods, and methodology. Behavioral Assessment, 10, 189-196.

Haynes, S., Leisen, M., \& Blaine, D. (1997). Design of individualized behavioral treatment programs using functional analytic clinical case models. Psychological Assessment, 9, 334-348.

Iwata, B., \& Worsdell, A. (2005). Implications of functional analysis methodology for the design of intervention programs. Exceptionality, 13, 25-35.

Kramer, T., Kleindorfer, K., \& Colarelly-Beatty, K. (1994). Who is the client: A replication and extension. Division of Consulting Psychology, 46, 11-18.

Loubat, M., \& Magaña, I. (2005). Centro de atención psicológica de la escuela de psicología en la Universidad de Santiago de Chile: Una experiencia de reestructuración inspirada en los modelos biopsicosocial y de salud pública. Revista de Psicología, 14, 47-62.

Martínez, P. (2006). Del motivo de consulta a la demanda en psicología. Revista de la Asociación Española de Psiquiatría, 26, 53-69.

Maschi, T., Schwalbe, C., Morgen, K., Gibson, S., \& Violette. N. (2009). Exploring the influence of gender in adolescents' service needs and service pathways. Children and Youth Services Review, 3, 257-264.

Muñoz-Martínez, A., \& Novoa-Gómez, M. (2010). Análisis de la confiabilidad y validación de un modelo de formulación clínica conductual. Tesis de Maestría no publicada, Pontificia Universidad Javeriana.

Mumma, G., \& Mooney, S. (2007). Incremental validity of cognitions in clinical case formulation: An intraindividual test in a case example. Journal of Psychopathology and Behavioral Assessment, 29, 17-28.

Mumma, G., \& Smith, J. (2001). Cognitive behavioral interpersonal scenarios: interformulator reliability and convergent validity. Journal of Psychophatology and Behavioral Assessment, 23, 203-221.

Northup, J., Wacker, D., Sasso, G., Steege, M., Cigrand K., Cook, J., \& Deraad, A., (1991). A brief functional analysis of aggressive and alternative behavior in an outclinic setting. Journal of Applied Behavior Analysis, 3, 509-522.

Organización Mundial de la Salud. (2005). Acción sobre los factores sociales determinantes de la salud: aprender de las experiencias anteriores. Extraído en mayo 02 de 2010, disponible en http://www. who.int/social determinants/en/

Ortega, M., Roca, G., Iglesias, M., \& Jurado, J. (2004). Pacientes hiperfrecuentadores de un centro de atención primaria: Características sociodemográficas, clínicas y de utilización de servicios sanitarios. Atención Primaria, 33, 78-85.

Porcel, M. (2005). El abandono en las terapias psicológicas. Aposta Revista de Ciencias Sociales, 14, 1-16.

Pradilla, A., Rueda, A., Salazar, L., Vélez, J., \& Muñoz, E. (2007). Determinantes en salud y políticas públicas: Identificación de indicadores de problemas de salud y factores asociados, basada en datos disponibles. Colombia Médica, 38, 151-262.

Quesada, S. (2004). Estudio sobre los motivos de consulta psicológica en una población universitaria. Universitas Psychologica, 3, 7-16.

Red de Instituciones de Servicios Universitarios de Atención Psicológica. (2007). Caracterización de la población que consulta a las ISUAP y sus motivos de consulta. Extraído en febrero 06 de 2010, disponible en www.ascofapsi.org.co/documentos/.../presentacion_encuentro.ppt.

Reep, A., \& Horner, R. (2000). Introducción al análisis funcional. En A. C. Reep \& R. H. Horner (Ed.), Análisis funcional de problemas de la conducta (pp. 1-5). Madrid: Paraninfo Thomson Learning.

Rodríguez, D. (2007). Motivos de consulta y clínica constructivista. Diversitas. Perspectivas en Psicología, 3, 239-247.

Solhan, M., Trull, T., Jahng, S., \& Wood, P. (2009). Clinical assessment of affective inestability: Comparing EMA indices questionnaire reports, and retrospective recall. Psychological Assessment, 21, 425-438.

Strauss, A., \& Corbin, J. (2002). Bases de la investigación cualitativa. Técnicas y procedimientos para desarrollar la teoría fundamentada. Medellín: Universidad de Antioquia.

Virués-Ortega, J., \& Haynes, S. (2005). Functional analysis in behavior therapy: Behavioral foundations and clinical application. International Journal of Clinical and Health Psychology, 5, 567-587. 